JURNAL

ADJECTIVISATION EN -ABLE/-IBLE DES VERBES

TROUVE DANS LE ROMAN LES CONFSSIONS PAR JEANJACQUES ROUSSEAU

disusun dan diajukan oleh:

MUTIARA MARSELINA SINURAT

NIM. 2123131034

Telah Diverifikasi Dan Dinyatakan Memenuhi Syarat untuk Diunggah pada Jurnal Online

Medan, November 2017

Menyetujui,
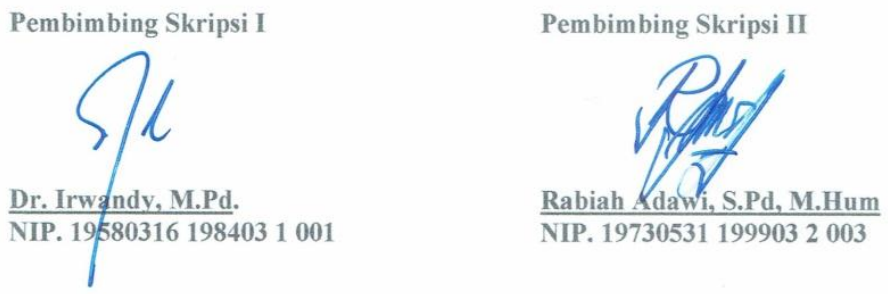

Editor Jurnal

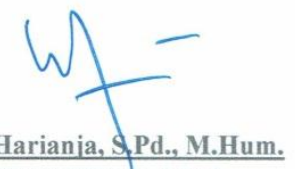

NIP. 197701212003122004

1 


\title{
ADJECTIVISATION EN -ABLE/-IBLE DES VERBES TROUVE DANS LE ROMAN LES CONFESSIONS PAR JEAN- JACQUES ROUSSEAU
}

\author{
Dr. Irwandy, M.Pd \\ Rabiah Adawi, S.Pd, M.Hum \\ Département des Langues Étrangères \\ Faculté des Lettres et des Arts \\ Université de Medan \\ Mèl : mutiaramarselinasinurat@gmail.com
}

\begin{abstract}
RÉSUMÉ
Mutiara Marselina Sinurat, 2123131034 "Adjectivisation En -Able/Ible Des Verbes Trouve Dans Le Roman Les Confessions Par Jean-Jacques Rousseau" Mémoire. Section Française, Département des Langues Étrangères, Faculté de Lettres et d'arts, Université de Medan. 2017.

Cette recherche a pour but de Savoir les adjectifs trouvés l'origine des verbes qui utilisent suffixe -able/-ible dans le Roman Les confessions JeanJacques Rousseau, Savoir les verbes étre formes en adjectif terminés par -able/ible dans le Roman Les confessions Jean-Jacques Rousseau et Connaître la signification en utilisent suffixe -able/-ible des verbes dans le Roman Les confessions Jean-Jacques Rousseau.

Pour analyser des données, prémièrement l'auteur chercher les adjectifs trouvés l'origine des verbes qui utilisent suffixe able/-ible dans le Roman Les confessions Jean-Jacques Rousseau. Ensuite, l'auteur Chercher les verbes étre formes en adjectif terminés par -able/-ible dans le Roman Les confessions JeanJacques Rousseau. Et puis, l'auteur décrivant la signification en utilisent suffixe able/-ible des verbes dans le Roman Les confessions Jean-Jacques Rousseau. Finalement, 1'auteur tire la conclusion.

Le résultat de cette recherche montre que, Les adjectifs qui sont trouvés l'origine des verbes qui utilisent suffixe -able dans le Roman Les confessions Jean-Jacques Rousseau sont 41 adjectifs. Les adjectifs qui sont trouvés l'origine des verbes qui utilisent suffixe -ible dans le Roman Les confessions Jean-Jacques Rousseau sont 10 adjectifs. Les verbes étre formes en adjectif terminés par-able/ible dans le Roman Les Confessions par Jean-Jacques Rousseau sont les verbes (
\end{abstract}


-er),(-ir),-(re -oir). La signification en utilisent suffixe -able/-ible des verbes dans le Roman Les Confessions par Jean-Jacques Rousseau qui exprime la qualité et exprime la maniére. Le résultat de cette recherche montre que le suffixe d'adjectif -able apparaît plus frequemment

Mots clés: adjectivisation, suffixes d'adjectif, roman les confessions par Jean Jacques Rousseau

\begin{abstract}
ABSTRAK
Mutiara Marselina Sinurat, 2123131034 “Adjectivisation En -Able/Ible Des Verbes Trouve Dans Le Roman Les Confessions Par Jean-Jacques Rousseau" Skripsi. Program Studi Pendidikan Bahasa Prancis, Jurusan Bahasa Asing, Fakultas Bahasa dan Seni, Universitas Negeri Medan. 2017.
\end{abstract}

Penelitian ini bertujuan untuk mengetahui kata sifat apa saja yang ditemukan dari kata kerja menggunakan sufiks -able/-ible dalam roman les confessions oleh Jean-Jacques Rousseau, mengetahui kata kerja apa saja yang dapat dibentuk menjadi kata sifat menggunakan sufiks -able/-ible, mengetahui makna apa yang ditemukan dengan menggunakan sufiks -able/-ible pada kata kerja.

Untuk menganalisis data, penulis terlebih dahulu mencari kata sifat yang yang ditemukan dari kata kerja menggunakan sufiks -able/-ible di dalam roman les confessions oleh Jean-Jacques Rousseau. Kemudian penulis menacari kata kerja - kata kerja yang dapat dibentuk menjadi kata sifat menggunakan sufiks able/-ible pada roman les confessions oleh Jean-Jacques Rousseau. Selanjutnya, penulis mendeskripsikan makna apa yang ditemukan dengan menggunakan sufiks able/-ible pada kata kerja dalam roman les confession oleh Jean-Jacques Rousseau. Terakhir, penulis dapat menarik kesimpulan.

Hasil penelitian menunjukkan bahwa kata sifat yang ditemukan dari kata kerja menggunakan suffiks -able dalam roman Les Confessions oleh Jean-Jacques Rousseau adalah 41 kata sifat. Kata sifat yang ditemukan dari kata kerja menggunakan suffiks -ible dalam roman Les Confessions oleh Jean-Jacques Rousseau adalah 10 kata sifat. Kata kerja yang dapat dibentuk menjadi kata sifat menggunakan suffiks -able/-ible dalam roman Les Confessions oleh Jean-Jacques Rousseau adalah kata kerja yang berakhiran (-er),(-ir),(-re,-oir). Makna yang ditemukan dengan penggunaan sufiks -able/-ible pada kata kerja di dalam roman Les Confessions oleh Jean-Jacques Rousseau adalah makna yang yang menjelaskan kualitas dan suatu cara. Hasil penelitian ini menunjukkan bahwa pemakaian sufiks -able yang sering muncul.

Kata kunci : adjectivalisasi, suffiks kata sifat, Roman Les Coonfessios oleh Jean-Jacques Rousseau 


\section{INTRODUCTION}

L'un des object de la recherche qui est intéressant à analyser est la formation du mot, parce qu'il se produit souvent dans une langue et aussi le processus de la morphologie. Morphologie a une étude de la langue (linguistique) qui examine la structure interne d'un lexique de mots ou de la langue. Le mot dans ce cas est considéré comme d'unités en forme et de sens qui montre les aspects de la valence syntaxique, à savoir les possibilités que ce mot à combiner avec les autres mots dans le groupe de mot. Au 19ème siècle, la morphologie du terme en tant que domaine linguistique compris que l'étude des changements systématiquement sur la forme du mot associé au sens. (Bauer, 1988:4)

La formation du mots est un objet qui est intéressant, aussi il y a beaucoup de problemes,donc, on peut doit chercher la solution systématiquement. l'un de type de la formation du mots est la formation de l'adjectif. exemple:

$$
\begin{aligned}
& \text { Adorer }(V)+- \text { Able }=\text { Adorable }(\text { Adj }) \\
& \text { Lire }(V)+- \text {-Ible }=\text { Lisible }(\text { Adj })
\end{aligned}
$$

Les mots au dessus n'est pas seulement analysé la forme du mots, mais aussi la function de l'unité en changer la forme du mots. Donc, dans le processus de la forme du mots, il se trouve deux types affixe, ces sont: les affixes inflexionnels et dérivationnelles. Affixes inflexionnels sont affixes qui peuvent générer formes un nouveau mot de lexsem essentiellement, alors que affixes dérivationnelles sont affixes qui génèrent de nouvelles lexem de base lexem. En d'autres termes, on peut dire que la morphologie inflexionnelle associé au processus d'affixation spécifié syntaxiquement, tandis que la mporphologie dérivationnelles utilisées pour former une nouvelle lexicale. (Bauer, 1988:80).

Ces deux processus morphologie, il devient intéressant d'étudier parce que le processus de formation des mots est certainement se produit dans toutes les langues et chaque langue montre différents processus. Dans cette étude, il parle de processus de dérivation, c'est l'adjectivisation. Ce terme fait référence à la processus de formation adjectif des autres catégories de mots comme verbes, noms, adverbiale en ajoutant des affixes dérivationnelles.

Ce sujet intéressant de discuter parce que l'adjectivisation a un rôle important dans l'utilisation de la langue, soit l'adjectivisation des verbes et des noms. Dans cette étude spécifiquement discuté Adjectivisation en -able / -ible sur le verbe dérivé des groups des verbes dans le roman les confessions par Jean Jacques-Rousseau. Les groupes des verbes en formation sont Les verbes en -er du $1^{\text {er }}$ groupe ont une ou deux et se conjuguent ao présent de l'indicatif avec e, es, e, ons, ez, en. Les verbes en -ir du $2^{\mathrm{e}}$ groupe comme finir se conjuguent au présent de l'indicatif à partir de la base fin- et des terminaisons is, is, it, issons, issez, issent. Les verbes en -ir, en -re, en -oir du $3^{\mathrm{e}}$ groupe ont une ou plusieurs bases et plusieurs types des terminaisons parce que n'est pas toutes les verbes peuvent changer devient l'adjectif avec l'utilisation suffixe -able/-ible. 


\section{RECOURS AUX THÉORIES}

\section{Morphosyntaxe}

Jean Dubois (1970:312) soutient que morphosyntaxe est la description des règles de combinaison des morphèmes pour former des mots, des syntagmes, et des phrase (morfosintaksis memaparkan mengenai aturan penggabungan kata untuk membentuk kata, sintagme, dan kalimat). Selon Harimurti Kridalaksana (1983:143) morfosintaksis merupakan struktur bahasa yang mencakup morfologi dan sintaksis sebagai satu organisasi atau kedua bidang itu tidak terpisahkan.

La morphologie fait partie de la science du langage pour discuter ou apprendre les subtilités vers certaines formes de mots, les significations et les fonctions des mots deux fonction grammaticale et syntaxique. Alors que la syntaxe elle-même fait partie de la linguistique qui étudie la relation entre la parole phrase une phrase de l'article avec les phrases de mots / phrases une autre clause, ou plus précisément d'étudier les subtilités phrases , clauses, les phrases, et le discours. Ainsi, un mot à la morphologie de la plus grande unité, la syntaxe est la plus petite unité .

(7) Ce train va vite. (KPI, $2007: 29)$

Le mot Ce est un morphème, le train représente également le morphème et ceux-ci sont inclus dans le domaine de l'étude de la morphologie. Ce pendant, si la relation entre Ce train comme sujet et comme un prédicat, il est inclus dans le champ d'étude de la syntaxe. Donc, on peut dire ici que le domaine de l'étude de la morphologie et de la syntaxeest un champ d'étude qui ne peuvent pas être séparés les un des autres

\section{Adjectif}

Dans le dictionnaire indonésien (2007 : 8-9) ajektiva adalah kata yang menerangkan nomina dan secara umum dapat bergabung dengan kata lain. Harimurti Kridalaksana (1983:3) berpendapat bahwa ajektiva merupakan kata yang menerangkan kata benda. Misalnya kata sèche (kering), dan blanche (putih). D'après Paul Robert (2001 :30) L'adjectif est un mot susceptible d'être adjoint directement (éphithète) ou indirectement (atribut) par l'intérmediane de quel ques verbes (être, notamment) au substantif avec lequel il s'accord pour exprimer un qualité (adjectif qualicatif, ou un adjectif determinatif, demonstratif, indefini, interrogatif, numerial, possesif, relatif). Certains adjectif prenent une signification differente selon qu'ilssont places avant ou après le nom. Les opinions sont presque aussi bien déclaré par Jean Dubois (1970:37) que l'adjectif est un mot qui qualifie ou détermine le substantif auquel il est joint. 


\section{Verbe}

Le verbe est un mot qui exprime, soit l'action faite, soit l'existence ou l'état du sujet ( Grevisse, 1993 :1337) Selon Harimurti Kridalaksana (1983: 226227) les verbes sont une classe de mots qui sert habituellement comme un prédicat, dans certains autres verbes de langue ont une des caractéristiques morphologiques caractéristiques telles que le temps, les aspects de la personnalité et le nombre. Une grande partie des verbes d'action représentent des éléments sémantiques, état, ou d'un processus. Il existe deux types de verbes sont des verbes transitif et intransitif, verbes transitifs désigne simplement l'idée que le verbe doit avoir un complement d'objet tandis que le verbe intransitif est un verbe qui n'a pas de complement d'objet.

Table 1.2 : les verbes sont classés en trois groupes

\begin{tabular}{|l|l|l|}
\hline $\mathbf{1}^{\text {er }}$ groupe & Verbes en - er $\rightarrow\left(\right.$ sauf aller : $3^{\mathrm{e}}$ gr. $)$ & $\begin{array}{l}\text { Aimer, Danser, étudier, former, habiter, se } \\
\text { promener, etc }\end{array}$ \\
\hline $\mathbf{2}^{\mathbf{e}}$ groupe & Verbes en -ir $\rightarrow$ & $\begin{array}{l}\text { Choisir, finir, garantir, grandir, obéir, } \\
\text { nourrir, réussir, etc. }\end{array}$ \\
\hline $\mathbf{3}^{\mathbf{e}}$ groupe & $\begin{array}{l}\text { Certains verbes en }- \text { ir } \rightarrow \\
\text { Verbes en }- \text { re } \rightarrow \\
\text { Et tous les verbes en }- \text { oir et -oire } \rightarrow\end{array}$ & $\begin{array}{l}\text { Dormir, haïr, offrir, ouvrir, tenir, venir, etc. } \\
\text { Attendre, faire, mettre, peindre, etc. } \\
\text { Devoir, pouvoir, savoir, voir, boire, croire, } \\
\text { etc. }\end{array}$ \\
\hline
\end{tabular}

\section{Adjectivisation}

L'Adjectivisation est la processus morphologie de formation adjectif des autres catégories de mots comme verbes, noms, adverbiale (Rohali, 2006: 60). Selon Jean Dubois (1970: 18) on appelle adjectivisation la transformation qui convertit un syntagme prépositionnel (prépositionnel suivi d'une syntagme nominal ) en un syntagme adjectival ou en adjectif . L'Adjectivisation peut être effectuée en dérivant une telles que les catégories lexicales de noms en adjectifs, verbes en adjectifs, ainsi que d'un adjectif en adjectif. L'adjectivisation peut être fait par affixation utilise des préfixes et des suffixes.

\section{Processus Morphologie}

La Structure Sur la base, un mot peut être classé en deux types, un mot ou des mots simples à savoir un mot morphème unique ou monomorfemis de morphèmes et a dit que plus d'un ou polimorfemis. Monomorfemis un mot qui ne connaîtra pas les événements de la formation morphème précédente, car il est le seul élément ou d'un membre en un mot. Versez la forme (pour) dans la phrase : 
- Il fait froid pour la saison

il fait froid pour lasaison est le mot, et ce mot est composé d'un morphème, soit morphème pour. La Morpheme pour être un écoulement pour n'a pas connu les événements de la formation. Cependant, contrairement à un mot polimorfemis. Les morphèmes qui sont membres de ce mot a vécu des événements de l'établissement précédent. Ces événements appellent habituellement la mise en place de processus morphologique ou morphématique.

Dérivationnel processus peut se faire de deux manières, à savoir la dérivation affixal (préfixes et suffixes) et non affiixal. Composition processus peut être divisé en deux façons de composition populaire et composition Savante (Rohali, 2006: 20) La composition est un processus populaire la formation des mots par l'ajout d'un mot avec un autre mot qui est Juxtapose. En d'autres termes, les deux éléments sont non-attachés et forment un nouveau mot avec un sens nouveau.

(1) Avoir un portefeuille bien garni (KPI, 2007 :807)

Dans l'exemple (1) ci-dessus sont mot formé à partir du portefeuille mot feuille et porte qui ont formé un nouveau mot avec un sens nouveau. La seconde est la composition Savante. La composition de type est une combinaison de deux mots latin ou le Grec la formation d'un nouveau mot avec un sens nouveau, si la composition populaire de deux lexiques formant une forme libre, le type de cette deuxième composition au contraire, est une forme de non libre.

(2) Les revolutions régicides (KPI, $2007: 890)$

Dans l'exemple (2), le mot régicides qui vient de la régir de base, qui a obtenu un suffixe $\{$-cide $\}$. Suffixe $\{$-cide $\}$ lui-même vient du suffixe latin $\{$-cida $\}$.

\section{Dérivation}

Harimurti Kridalaksana (1993 : 40) mengemukakan bahwa derivasi adalah proses pengimbuhan afiks non inflektif pada kata dasar untuk membentuk kata, Pendapat ini hampir sama dalam Kamus besar bahasa Indonesia (2007 : 256 432) bahwa derivasi adalah pengimbuhan afiks yang tidak bersifat infleksi pada bentuk dasar untuk membentuk kata. Selon Khusnin (www. Morfologi-bahasa Indonesia.com), berpendapat bahwa derivasi adalah prosede morfologis yang menghasilkan kata-kata yang makna leksikalnya berbeda dari kata pangkal pembentuknya.

Tandis que Grevissse (1993:197) la dérivation est l'opération par laquelle on crée une nouvelle unitélexicale en ajoutant à un mot existant un élément non autonome ou affixe.

(1) Un vrai Renoir (KPI, $2007: 1110$ )

2) Il sait reconnaitre le vrai du faux. (KPI, 2007 :1110) 
Dans l'exemple (1) au-dessus duquel il y a un mot vrai un adjectif, le mot vrai est de la classe modifiée il est devenu un nom dans l'échantillon (2), mais dans amendement dit vrai ne change pas parce qu'il n'a pas imprégné pour fixer tout type, mais la classe qu'il a changé.

D’apres Hamon (1983:36) Pour former des dérivés, le française posséde de nombreux suffixes, de formation populaire ou de formation savante : Suffixes de noms, d'adjectifs, des verbes, d'adverbes, mot latins ou grecs jouant rôle de suffixes. Le suffixes ajoute un sens nouveau à celui du raical, qui par ailleurs peut voir sa forme altérée :

- moule, moul - ure (chute du e final du radical )

- bras, bras-s-ée (redoublement de la consonne finale du radical )

- main, men-ottre ( altération du radical)

- bijou, bijou-t-ier ( addition d'une conssone)

\section{Suffixes d'adjectifs}

Ils servent à former des adjectifs. Les principaux suffixes d'adjectifs sont: -able, -ible, -uble, -ain, -aire, -al, -ard, -âtre, -el, -eux, -eur, -esque, -if, -ique, -iste, -ois, -aud, -et, -elet, -in, -ot, -u, -é. Par exemple: aim-able, aud-ible, sol-uble, proch-ain, simil-aire, fin-al, vant-ard, bleu-âtre, mort-el, parees-eux, flatt-eur, livr-esque, malad-if, poét-ique, social-iste, brest-ois, lourd-aud, propr-et, aigrelet, argent-in, pâl-ot, barb-u, ail-é. Ces principaux suffixes d'adjectifs donnent aux mots obtenus divers sens: possibilité, origine, qualité, sens péjoratif, diminutif, etc. Par exemple, audible : qu'on peut ettendre; barbu: pourvu de barbe; pâlot: un peu (trop) pâle (diminutif et péjoratif).

D'apres Michaela Chateaux dans le site http://ameliorersonfrancais.com, able et -ible : Les suffixes -able et -ible construisent des adjectifs à partir de bases nominales et verbales. Ils expriment une manière d'être, ex. Charitable et Paisible, ou quelque chose qui peut être ce à quoi réfère le nom ou le verbe sur lequel il travaille, ex. buvable et lisible.

\section{MÉTHODOLOGIE DE LA RECHERCHE}

La méthode de recherche est très importante dans une recherche afin que le résultat obtenu soit satisfaisant. Avec la méthode de recherche correcte, les problèmes apparus dans la recherche peuvent être résous et le cherceur peut trouver les variétés, si bien que les données de l'analyse peuvent être bien dirigées. Le processus de recheche qui est orientée va produire l'information et l'analyse de données. Cette information peut être acceptée naturellement par tout le monde.

Selon Santoso dans Herawani (2016 : 4), metodologi adalah suatu cara yang masuk akal atau ilmiah dalam mencari kebenaran keilmuwan. c'est-à-dire une méthodologie considère une manière avec avertie ou scientifique pour découvrir la vérité de la recherche.

Dans cette recherche l'auteur utilise la méthode qualitative. Selon Mulyana (2001 : 178) metodologi kualitatif sebagai prosedur penelitian yang 
menghasilkan data deskriptif berupa kata-kata tertulis atau lisan dari orangorang dan perilaku yang dapat diamati. C'est-à-dire la données qualitative est une procédure de recherche qui produit la donné descriptive et se compose par des mot écrits ou orales des personnes et les attitudes qui peuvent être observées. La technique du collecte de données est la metode simak. Selon Sudaryanto (1993 :192) la metode simak est une méthode qui est faite en regardant ou observant l'utilisation de langue. C'est à dire la metode simak est une méthode d'étude du document. La technique de base qui est utilisée dans ce cas est la teknik sadap, c'est-à-dire l'auteur va copier la donnée sous la forme écrit. Ensuite la technique avancée tilisée est la teknik catat, c'est-à-dire l'auteur écrite tous les données qui sont pertinent à la recherche.

Les procédures d'analyse de données dans cette recherche se composent de :

1. Lire le roman les confessions par Jean-Jacques Rousseau

2. Chercher les adjectifs trouvés l'origine des verbes qui utilisent suffixe able/-ible dans le Roman Les confessions Jean-Jacques Rousseau.

3. Souligner les adjectifs trouvés l'origine des verbes qui utilisent suffixe -able/-ible dans le Roman Les confessions Jean-Jacques Rousseau.

4. Chercher les verbes étre formes en adjectif terminés par-able/ible dans le Roman Les confessions Jean-Jacques Rousseau.

5. Faire le tableau sur les adjectifs trouvés l'origine des verbes qui utilisent suffixe able/-ible dans le Roman Les confessions Jean-Jacques Rousseau.

6. Décrivant la signification en utilisent suffixe -able/-ible des verbes dans le Roman Les confessions Jean-Jacques Rousseau.

7. Faire la conclusion.

\section{RÉSULTAT DE LA RECHERCHE}

Les suffixes d'adjectif peuvent server à former des adjectifs. Les principaux suffixes d'adjectifs donnent aux mots obtenus, divers sens: qualité, et manière. Et cette recherche va donner les explications sur des suffixes d'adjectif. Voici la liste des suffixes d'adjectif qui sont server à former des verbes en adjectif (-Able, -Ible).

Récapitulatif Des suffixes d'adjectif qui sont-Able/-Ible dans le roman.

\begin{tabular}{|l|l|l|}
\hline No & Suffixes d'adjectif & Quantité \\
\hline 1. & -able & 41 \\
\hline 2. & -ible & 10 \\
\hline
\end{tabular}

\section{ANALYSE DE DONNÉES DE LA RECHERCE}

Les données d'identification à base verbes constitutifs sont des verbes -er, -ir, -re décrit dans le chapitre précédent. Le tableau suivant expose le suffixe qui 
signifie de nouveaux l'adjectifs utilisés alors analyser les types de verbes, ils sont plus faciles à déterminer.

Les suffixes d'adjectif -Able dérivé des verbes -er

\begin{tabular}{|c|c|c|}
\hline $\begin{array}{l}\text { Suffixes } \\
\text { d'adjectifs }\end{array}$ & $\begin{array}{l}\text { Lignes des suffixes } \\
\text { d'adjectifs -able }\end{array}$ & $\begin{array}{l}\text { Lignes des suffixes d'adjectifs -able } \\
\text { dérivé des verbes -er }\end{array}$ \\
\hline \multirow{34}{*}{-able } & 1. aimable & 1. aimer \\
\hline & 2. agréable & 2. agréer \\
\hline & 3. admirable & 3. admirer \\
\hline & 4. détestables & 4. détester \\
\hline & 5. louable & 5. louer \\
\hline & 6. estimable & 6. estimer \\
\hline & 7. raisonnable & 7. raisonner \\
\hline & 8. vénérable & 8. vénérer \\
\hline & 9. coupable & 9. couper \\
\hline & 10. imaginable & 10. imaginer \\
\hline & 11. honorable & 11. honorer \\
\hline & 12. respectable & 12. respecter \\
\hline & 13. portable & 13. porter \\
\hline & 14. effroyable & 14. effrayer \\
\hline & 15. adorable & 15. adorer \\
\hline & 16. insupportable & 16. supporter \\
\hline & 17. inexprimables & 17. exprimer \\
\hline & 18. inévitable & 18.éviter \\
\hline & 19. indéchiffrables & 19.déchiffrer \\
\hline & 20. inaltérable & 20. altérer \\
\hline & 21. Défavorable & 21. défavoriser \\
\hline & 22. impayable & 22. payer \\
\hline & 23. inséparable & 23. séparer \\
\hline & 24. inébranlable & 24. ébranler \\
\hline & 25. pitoyable & 25. apitoyer \\
\hline & 26. désagreable & 26. agréer \\
\hline & 27. méprisable & 27. mépriser \\
\hline & 28. indomptable & 28.dompter \\
\hline & 29. semblable & 29. sembler \\
\hline & 30. indénombrable & 30. dénombrer \\
\hline & 31. inestimable & 31. estimer \\
\hline & 32. inséparable & 32. séparer \\
\hline & 33. inépuisable & 33. épuiser \\
\hline & 34. insurmontable & 34. surmonter \\
\hline
\end{tabular}


1. Une soeur de mon père, fille aimable et sage, prit si grand soin de moi, qu'elle me sauva. (les confessions 1782:6)

Aimable $($ adj $)=$ aimer + -able

Dans cette phrase au dessus le mot "aimable" est un adjectif. Il vient de "aimer" (verbe -er) + -able (suffixe). Le suffixe -Able construit des adjectifs à partir de bases verbales. La signification en utilisent suffixe -able de verbe exprime la qualité . La qualité de la sœur de mon père est aimable est sage. (page 32)

Les suffixes d'adjectif -Able dérivé des verbes -ir

\begin{tabular}{|l|l|l|}
\hline $\begin{array}{l}\text { Suffixes } \\
\text { d'adjectifs }\end{array}$ & $\begin{array}{l}\text { Lignes des suffixes } \\
\text { d'adjectifs -able }\end{array}$ & $\begin{array}{l}\text { Lignes des suffixes d'adjectifs -able } \\
\text { dérivé des verbes -ir }\end{array}$ \\
\hline \multirow{2}{*}{-able } & 1. haïssable & 1. haïr \\
\cline { 2 - 3 } & 2. convenable & 2. convenir \\
\cline { 2 - 3 } & 3. affable & 3. affaiblir \\
\hline
\end{tabular}

1. je ne savais porter mes désirs que vers l'espèce de volupté qui m'était connue, sans aller jamais jusqu'à celle qu'on m'avait rendue haïssable. (les confession 1782:16)

$$
\text { haïssable }(\operatorname{adj})=\text { haïr }+ \text {-able }
$$

Dans cette phrase au dessus le mot "haïssable" est un adjectif. Il vient de "haï" (verbe -ir) + -able (suffixe). Le suffixe -able construit des adjectifs à partir de bases verbales. La signification en utilisent suffixe -able de verbe exprime le maniére. (page 45)

Les suffixes d'adjectif -Able dérivé des verbes (-re,-ir, -oire,-oir)

\begin{tabular}{|l|l|l|}
\hline $\begin{array}{l}\text { Suffixes } \\
\text { d'adjectifs }\end{array}$ & $\begin{array}{l}\text { Lignes des suffixes } \\
\text { d'adjectifs -able }\end{array}$ & $\begin{array}{l}\text { Lignes des suffixes d'adjectifs -able } \\
\text { dérivé des verbes -re }\end{array}$ \\
\hline \multirow{4}{*}{-able } & 1. croyable & 1. croire \\
\cline { 2 - 3 } & 2. ignoble & 2. ignore \\
\cline { 2 - 3 } & 3. inconcevable & 3. concevoir \\
\cline { 2 - 3 } & 4. incroyable & 2. croire \\
\hline
\end{tabular}

1. l'espoir de gagner force écus par une voie ignoble flattait peu mon humeur hautaine (les confession 1782:30)

$$
\text { ignoble }(\operatorname{adj})=\text { ignorer }+ \text {-able }=
$$

Dans cette phrase au dessus le mot "ignoble" est un adjectif. Il vient de "ignorer" (verbe) + -able (suffixe). Le suffixe -able construit des adjectifs à partir de bases verbales. La signification en utilisent suffixe -able de verbe exprime le qualité. (page 47)

Les suffixes d'adjectif -ible dérivé des verbes -er

\begin{tabular}{|l|l|l|}
\hline Suffixes & Lignes des suffixes & Lignes des suffixes d'adjectifs -ible \\
\hline
\end{tabular}




\begin{tabular}{|l|l|l|}
\hline d'adjectifs & d'adjectifs -ible & dérivé des verbes -er \\
\hline \multirow{4}{*}{ - ible } & 1. sensible & 1. sensibiliser \\
\cline { 2 - 3 } & 2. horrible & 2. horrifier \\
\cline { 2 - 3 } & 3. impossible & 3. imposer \\
\cline { 2 - 3 } & 4.inflexible & 4. infléchir \\
\cline { 2 - 3 } & 5. insensible & 5. insensibiliser \\
\cline { 2 - 3 } & 6. possible & 6. possibiliser \\
\hline
\end{tabular}

1. De tous les dons que le Ciel leur avait départis, un coeur sensible est le seul qu'ils me laissèrent ; mais il avait fait leur bonheur, et fit tous les malheurs de ma vie. (les confession 1782:6)

$$
\text { sensible }(\operatorname{adj})=\text { sensibiliser }+ \text {-ible }
$$

Dans cette phrase au dessus le mot "sensible" est un adjectif. Il vient de "sensibiliser" (verbe) + -ible (suffixe). Le suffixe -ible construit des adjectifs à partir de bases verbales. La signification en utilisent suffixe ible de verbe exprime le qualité. (page 49)

Les suffixes d'adjectif -ible dérivé des verbes -ir

\begin{tabular}{|l|l|l|}
\hline $\begin{array}{l}\text { Suffixes } \\
\text { d'adjectifs }\end{array}$ & $\begin{array}{l}\text { Lignes des suffixes } \\
\text { d'adjectifs -ible }\end{array}$ & $\begin{array}{l}\text { Lignes des suffixes d'adjectifs -ible } \\
\text { dérivé des verbes -ir }\end{array}$ \\
\hline \multirow{4}{*}{-ible } & 1. faible & 1. faiblir \\
\cline { 2 - 3 } & 2. terrrible & 2. terrir \\
\cline { 2 - 3 } & 3. susceptible & 3. suscrir \\
\cline { 2 - 3 } & 4. inflexible & 4. infléchir \\
\hline
\end{tabular}

1. C'était un grand garçon fort efflanqué, fort fluet, aussi doux d'esprit que faible de corps, et qui n'abusait pas trop de la prédilection qu'on avait pour lui dans la maison comme fils de mon tuteur

(les confession 1782:12)

Faible $(\operatorname{adj})=$ faiblir + -ible

Dans cette phrase au dessus le mot "faible" est un adjectif. Il vient de "faiblir" (verbe) + -ible (suffixe). Le suffixe -ible construit des adjectifs à partir de bases verbales. La signification en utilisent suffixe -ible de verbe exprime le qualité. (page 51)

\section{CONCLUSION}

1. Les adjectifs qui sont trouvés l'origine des verbes qui utilisent suffixe -able dans le Roman Les confessions Jean-Jacques Rousseau sont 41 adjectifs : 
aimable, agréable, admirable, détestable, louable, estimable, raisonnable, vénérable, coupable, imaginable, honorable, respectable, portable, effroyable, haïssable, convenable, affable, croyable, ignoble, inconcevable, incroyable.

Les adjectifs qui sont trouvés l'origine des verbes qui utilisent suffixe -ible dans le Roman Les confessions Jean-Jacques Rousseau sont 10 adjectifs : sensible, horrible, impossible, inflexible, insensible, possible, faible, terrible, susceptible, inflexible.

2. Les verbes étre formes en adjectif terminés par-able/-ible dans le Roman Les Confessions par Jean-Jacques Rousseau sont :

- Les verbes -er sont aimer, agréer, admirer, détester, louer, estimer, raissoner, vénérer, couper, imaginer, honorer, respecter, porter, effrayer, supporter, vérité, louer, couper, payer, éviter.

- Les verbes -ir sont haïr, convenir, affaiblir

- Les verbes -re,-ir,-oir sont croire, ignore, concevoir, croire.

Les verbes sont qui formés les adjectifs terminés par le suffixe -ible dans le roman Les Confessions Par Jean Jacques Rousseau sont :

- Les verbes -er sont sensibiliser, horrifier, imposer, insensibiliser, possibiliser

- Les verbes -ir sont faiblir, terrir, suscrir, infléchir

- Les verbes qui ne peuvent pas être formé les adjectifs en utilisant le suffixe -able/-ible sont les verbesirréguliers : -avoir, -être, -aller

3. La signification en utilisent suffixe -able/-ible des verbes dans le Roman Les Confessions par Jean-Jacques Rousseau qui exprime la qualité et exprime la maniére.

\section{BIBLIOGRAPHIE}

Alwi, Hasan. 2007. Kamus Besar Bahasa Indonesia. Jakarta : PT Gramedia Pustaka Umum

Arifin, dkk. 1991. Kamus Prancis Indonesia. Jakarta : PT Gramedia Pustaka Utama

Bescherelle. 1997. La grammaire pour tous. Paris : Haitier

Dubois, Jean dan François. 1970. Eléments de linguistique français : syntaxe. Paris : Librairie Larousse.

Grevisse, 1993. Le Bon Usage : Grammaire Français, Treizième édition. Paris : Duculot

Hamon, Albert. 1983. Grammaire Pratique. Medan : FBS UNIMED

Kridalaksana, Harimurti. 1983. Kamus Linguistik. Jakarta : PT Gramedia Pustaka Utama 
Mahsun. 2011. Metode penelitian Bahasa. Jakarta : PT Raja Grafindo Persada

Michael Robert, Jean dan Isabelle-Chollet. 2009. Précis de Grammaires. Paris: CLE international

Muslich, Masnur. 2008. Tata Bentuk Bahasa Indonesia. Jakarta : PT Gramedia Pustaka Utama

Mulyana. 2001. Metode Penelitian Kualitatif Dan Kuantitatif . Bandung : PT.Remaja

Parera, Daniel. 2007. Morfologi. Jakarta : PT Gramedia Pustaka Utama

Pujiastuti, Anik. 2011. Ajektivalisasi kategori verba dalam bahasa perancis. Yoyakarta : Universitas Negeri Yogyakarta. Mémoire

Ramlan, M. 1987. Morfologi: suatu tinjauan deskriptif. Yogyakarta : C.V Karyono.

Robert, Paul. 2001. Le Grand Robert de la Langue Français. Paris :

Dictionnaires le Robert

Rousseau, Jean-Jacques. 1782. Les Confessions. Jakarta : PT Gramedia Pustaka Utama.

Rohali. 2006. Morfologi Bahasa Perancis. Yogyakarta: FBS UNY

Santoso, Joko. 2006. Semantik. Yogyakarta : FBS UNY

Sudaryanto. 1993. Metode dan aneka teknik Analisis Bahasa. Yogyakarta :Duta Wacana University Press

\section{SITOGRAPHIE}

http://www.academia.edu/3405921/Kajian-morfologi-derivasional-daninfleksional-dalam-bahasa-indonesia

http://sastra.um.ac.id/wp-content/uploads/2009/10/Fungsi-dan-Makna-AfiksInfleksi-pada-Verba-Afiksasi-Bahasa-Indonesia-Ermanto.pdf

http://www.edsc.gc.ca/fra/emplois/ace/docs/outils/cahier_vocabulaire.pdf

https://fr.wiktionary.org/wiki/-able

http://www.cnrtl.fr/definition/-able

http://www.ac.grenoble.fr/ecole/74/maitriselangue74/IMG/pdf/Differences

Lexique Vocabulaire.pdf

$\underline{\text { www.assistancescolaire.com/eleve/6e/francais/lexique/R-roman-fc_r05 }}$ 